\title{
Epidemiology of pancreatic carcinoma in Canada: 1931 to 1985
}

\author{
ANTHONY A YIOMAMITIS, BSc, BA, BMATH, BES, MSc, MHSC, MED
}

\begin{abstract}
Pancreatic carcinoma accounts for approximately one of every 20 deaths from cancer and one out of every 30 new cases of cancer in Canada. It is among the 10 leading causes of death from cancer and the 10 leading sites of newly diagnosed cases for a number of age groups. Age standardized mor tality rates have risen sharply in both males ( 2.0 to 9.5 deaths per 100,000 population per year) and females ( 2.2 to 5.7 deaths per 100,000 population per year) during 1931 to $1985(\mathrm{P}<$ 0.0001 ). The increase in standarized rates, 0.14 and 0.07 additional deaths per 100,000 population per year in males and females, respectively, is attributable to significant increases in age specific rates for males and females aged 35 to 44,45 to 54,55 to 64 , 65 to 74.75 to 84 and over 85 years old $(\mathrm{P} \leq 0.0005)$ in whom rates have risen by as much as 2.5 additional deaths per 100,000 population per year. Although age standardized incidence rates have risen marginally in males $(P=0.085)$, age specific rates in males aged 0 to 24 years have risen significantly $(P=0.01)$. In contrast, standarized incidence rates have risen sharply in females ( 0.12 additional new cases per 100,000 population per year; $\mathrm{P}=0.0007$ ), which was also characteristic of age specific rates for women aged 45 to 54,55 to 64,65 to 74 and 75 to 84 years old $(\mathrm{P}<0.03)$ where rates have risen by 0.15 to 1.65 additional new cases per 100,000 population per year. Recent age specific incidence and mor tality rates indicate that morbidity and mortality rates rise sharply after age 45 , when rates double between successive 10-year age groups, and peak for males and females aged more than 85 years. Can J Gastroenterol $1988 ; 2(2): 71-74$
\end{abstract}

Key Words: Cancer, Morbidity, Mortality, Pancreas, Rates, Trends

Correspondence and reprints: Dr A. Ayiomamitis, PO Box 512, Station P. Toronto, Ontario M5S 2T1 Recelved for publication December 18, 1987. Accepted May 2, 1988
$\mathrm{P}$ ANCREATIC CARCINOMA WAS REsponsible for 2420 deaths in Canada during 1985 and was diagnosed in another 2447 Canadians during 1982 (1-2). It accounts for approximately $5.1 \%$ and $5.4 \%$ of all deaths from cancer in males and females, respectively, and about $3.2 \%$ and $2.7 \%$ of all newly diagnosed cases of cancer.

Carcinoma of the pancreas is ranked among the 10 leading sites of newly diagnosed cases of cancer in males aged 45 to 49 and 50 to 54 and males and females aged 55 to 59,60 to 64,65 to 69 . 70 to 74,75 to 79,80 to 84 and 85 years and older. It represents the seventh leading site in males and the 10th leading site in females for the incidence of cancer (3).

Mortality from pancreatic carcinoma is ranked as high as the fourth leading cause of death from cancer for a number of age groups in males and females. It is among the 10 leading causes of death from cancer in males aged 35 to 39 and 
males and females aged 40 to 44,45 to 49,50 to 54,55 to 59,60 to 64,65 to 69 , 70 to 74,75 to 79,80 to 84 and 85 years and older and accounts for as many as $7.2 \%$ of all deaths due to cancer (3). The lifetime probability of dying from this neoplastic disease in Canada is $1.27 \%$ for males and $1.23 \%$ in females, (unpublished data).

Projections by the Canadian Cancer Society indicate that approximately 2600 Canadians will die from pancreatic carcinoma in 1987 and this neoplastic disease will be diagnosed in another 2600 during that year.

Over 3750 hospital admissions during 1982 were for the diagnosis of this neoplasm ( 17.2 admissions in males and 13.4 admissions in females per 100,000 population per year). Admission rates were least in males and females under the age of 45 , below 5 admissions per 100,000 population per year. However, rates rose quickly with increasing age and were greatest in males and females aged 75 to 84 and 85 years and older where they exceeded 150 and 75 admissions per 100,000 population per year, respectively. The mean hespital stay was 20.8 and 25.1 days per admission for males and females, respectively (3).

Statistics on morphology indicate that $36 \%$ of all male cases are adenocarcinomas, $19 \%$ are epithelial neoplasms (not otherwise specified), $7.6 \%$ other specified types and the remaining $37.3 \%$ are listed as unspecified histology (3). Figures for females are similar, namely $36 \%$, $20 \%, 1.9 \%$ and $36.7 \%$, respectively, with cystic, mucinous and serous neoplasms accounting for the remaining $5.1 \%$.

The epidemiology of pancreatic carcinoma has been described for Australia (4), England and Wales (5) and Japan (6). However, similar detailed analyses based on Canadian data have been lacking and the purpose of this study was to address this deficiency.

\section{MATERIALS AND METHODS}

A database consisting of the annual number of reported new primary cases and deaths from pancreatic carcinoma as well as estimates of mid-year populations was assembled using the annual publications of Statistics Canada (1-2) and the rubrics of the International Clas- sification of Diseases (ninth revision). Data were collected by age group ( 0 to 24,25 to 34,35 to 44,45 to 54,55 to 64 , 65 to 74,75 to 84 and 85 years and older) and sex for the periods 1970-82 (morbidity figures) and 1931-85 (mortality figures).

Simple bivariate plots of age specific and age standardized morbidity (or incidence) and mortality rates as a function of calendar year indicated that linear regression analysis was suitable for the assessment of secular trends as both age specific and age standardized rates did not demonstrate any curvilinear relationships. Age specific and age standardized incidence and mortality rates were used as the dependent variable in the regression analysis whereas the year from 1970 (morbidity rates) and from 1931 (mortality rates) was used as the independent variable, thus allowing for the estimation of the significance of annual rates of change in these rates. All parametric analyses were confirmed using Spearman's (nonparametric) rank-order correlation coefficient. Statistics with associated levels of significance of 0.05 or less were deemed significant.

Other details regarding data manage- ment and statistical analysis, the definition of death used by Statistics Canada, the age at death provided, and the type of census enumeration used to derive mid-year population estimates are described elsewhere $(7,8)$.

\section{RESULTS}

Age standarized morbidity rates (ASMRs): Standarized morbidity rates for males during 1970-82 have consistently exceeded corresponding rates in females by $49 \%$ to $88 \%$ (Table 1). Rates for males have generally fluctuated between 6.8 and 8 new cases per 100,000 population per year. The only exceptions were 1981 and 1982 where rates rose to almost 10 new cases per 100,000 population per year. Rates in females have risen from approximately 4.5 to nearly 6 new cases per 100,000 population per year during 1970-82. Whereas the trend towards higher ASMRs in males is marginally significant, about 0.11 additional new cases per 100,000 population per year $(\mathrm{P}=0.085)$, the increase in female standardized incidence rates is highly significant $(0.12$ additional new cases per 100,000 population per year; $\mathrm{P}=0.0007$ ) (Table 2).

\section{TABLE 1}

Age standardized morbidity (ASMR) and mortality (ASDR) rates in Canada for selected years during 1931 to 1985

\begin{tabular}{|c|c|c|c|c|c|c|}
\hline \multirow[b]{2}{*}{ Year } & \multicolumn{3}{|c|}{ ASMR } & \multicolumn{3}{|c|}{ ASDR } \\
\hline & Male & Female & $\mathrm{M} / \mathrm{F}$ & Male & Female & $\mathrm{M} / \mathrm{F}$ \\
\hline 1931 & - & - & - & 2.08 & 2.23 & 0.93 \\
\hline 1936 & - & - & - & 3.17 & 2.59 & 1.22 \\
\hline 1941 & - & - & - & 4.44 & 3.74 & 1.19 \\
\hline 1946 & - & - & - & 4.94 & 3.12 & 1.58 \\
\hline 1951 & - & - & - & 6.12 & 3.70 & 1.65 \\
\hline 1956 & - & - & - & 6.78 & 4.62 & 1.47 \\
\hline 1961 & - & - & - & 7.95 & 4.49 & 1.77 \\
\hline 1966 & - & - & - & 8.58 & 4.90 & 1.75 \\
\hline 1970 & 7.86 & 4.47 & 1.76 & 9.53 & 5.05 & 1.89 \\
\hline 1971 & 7.95 & 4.23 & 1.88 & 9.18 & 5.10 & 1.80 \\
\hline 1972 & 7.61 & 4.61 & 1.65 & 9.07 & 5.44 & 1.67 \\
\hline 1973 & 7.51 & 4.75 & 1.58 & 9.51 & 5.50 & 1.73 \\
\hline 1974 & 6.83 & 4.06 & 1.68 & 8.69 & 5.35 & 1.62 \\
\hline 1975 & 7.24 & 4.69 & 1.54 & 8.21 & 5.52 & 1.49 \\
\hline 1976 & 6.89 & 4.42 & 1.56 & 8.98 & 5.46 & 1.64 \\
\hline 1977 & 7.01 & 4.68 & 1.50 & 8.73 & 5.54 & 1.58 \\
\hline 1978 & 7.48 & 5.03 & 1.49 & 9.00 & 5.60 & 1.61 \\
\hline 1979 & 7.99 & 5.09 & 1.57 & 9.36 & 5.55 & 1.69 \\
\hline 1980 & 7.64 & 4.98 & 1.53 & 8.61 & 5.42 & 1.59 \\
\hline 1981 & 8.99 & 5.91 & 1.52 & 8.57 & 5.58 & 1.54 \\
\hline 1982 & 9.77 & 5.85 & 1.67 & 9.35 & 5.49 & 1.70 \\
\hline 1983 & - & - & - & 8.90 & 5.67 & 1.57 \\
\hline 1984 & - & - & - & 8.52 & 5.56 & 1.53 \\
\hline 1985 & - & - & - & 8.58 & 5.53 & 1.55 \\
\hline
\end{tabular}

Rates are per 100.000 of world standard population 
TABLE 2

Annual rates of change in age specific and age standardized morbidity rates by age group and sex during 1970-82

\begin{tabular}{lccccc}
\hline \multirow{2}{*}{$\begin{array}{l}\text { Age group } \\
\text { (years) }\end{array}$} & \multicolumn{2}{c}{ Male } & & \multicolumn{2}{c}{ Female } \\
\cline { 2 - 3 } \cline { 5 - 6 } & $\begin{array}{c}\text { Rate } \\
\text { of change }\end{array}$ & Pvaluet $^{*}$ & & $\begin{array}{c}\text { Rate } \\
\text { of change }\end{array}$ & Pvaluet $^{*}$ \\
\hline $00-24$ & +0.004 & 0.013 & & 0.000 & 0.98 \\
$25-34$ & +0.006 & 0.54 & & -0.006 & 0.40 \\
$35-44$ & -0.019 & 0.51 & & -0.016 & 0.54 \\
$45-54$ & +0.085 & 0.46 & & +0.152 & 0.026 \\
$55-64$ & +0.321 & 0.14 & & +0.343 & 0.008 \\
$65-74$ & +0.751 & 0.18 & & +0.976 & 0.002 \\
$75-84$ & +1.494 & 0.10 & & +1.647 & 0.0007 \\
$85+$ & +2.000 & 0.28 & & +0.212 & 0.82 \\
ASMR & +0.106 & 0.085 & & +0.116 & 0.0007 \\
\hline
\end{tabular}

- Per 100,000 of population per year, $+P$ value associated with the null hypothesis that rate of change is O. ASMR Age standardized morbidity rate

The stability of ASMRs noted for males is characteristic of the seven age specific rates studied which did not increase or decrease significantly during the period studied $(P \geq 0.10)$. However, rates in males increased significantly during this period $(\mathrm{P}=0.013$ ) (Table 2$)$. In contrast, the rise in female ASMRs is associated with corresponding significant increases in age specific rates for females aged 45 to 54,55 to 64,65 to 74 and 75 to 84 years in whom rates increased by 0.15 to 1.65 additional new cases per 100,000 population per year since 1970 $(\mathrm{P}<0.03)$ (Table 2$)$.

Age standarized mortality rates (ASDRs): The increase in ASDRs was dramatic in both males and females ( $\mathrm{Ta}$ ble 1) where rates increased from approximately 2 to as many as 9.4 deaths per 100,000 population per year in males during 1931 to 1985 and from about 2 to slightly under 6 deaths per 100,000 population per year in females during the same period. The significant rise in ASDRs for both males, 0.14 additional deaths per 100,000 population per year, and females, 0.07 additional deaths per 100,000 population per year, is highly significant $(\mathrm{P}<0.0001)$ (Table 3$)$.

Examination of age-sex-specific mortality rates indicates that the appreciable rise in male ASDRs is attributable to significant increases in mortality in males aged 35 to 44,45 to 54,55 to 64,65 to 74,75 to 84 and 85 years and older where rates increased by as much as 2.5 additional deaths per 100,000 population per year $(\mathrm{P} \leq 0.0005)$. Similar results were noted in females as rates for the same six age groups rose significantly $(0.01$ to 1.8 additional deaths per 100,000 population per year; $\mathrm{P}<0.0001$ ).

As was the case with morbidity rates, standardized mortality rates were consistently higher in males and they generally exceeded rates in females by $50 \%$ to $90 \%$.

The rapid increase in morbidity and mortality as a function of age is illustrated in Table 4 where rates for the most recent years for which data are available are presented. Morbidity was well below 5 new cases per 100,000 population per year in males and females under the age of 45 . However, rates rose rapidly thereafter, approximately doubling between successive 10-year age groups and plateauing for those aged 85 years and older where rates peaked at 119 and 88 new cases per 100,000 population per year.

A similar pattern was noted for mortality rates which were well below 3 deaths per 100,000 population per year in males and females under the age of 45. Mortality rates increased rapidly after age 45 , approximately doubling between successive 10-year age groups, and peaking for males and females aged 85 years and older ( 133 and 83 deaths per 100,000 population per year in males and females, respectively). The similarity between morbidity and mortality rates suggests that treatment is not very effective and/or diagnosis is often late.

\section{TABLE 3}

Annual rates of change in age specific and age standardized mortality rates by age group and sex during 1931-85

\begin{tabular}{|c|c|c|c|c|}
\hline \multirow[b]{2}{*}{ Age group (years) } & \multicolumn{2}{|c|}{ Male } & \multicolumn{2}{|c|}{ Female } \\
\hline & $\begin{array}{c}\text { Rate } \\
\text { of change* }\end{array}$ & Pvaluet & $\begin{array}{c}\text { Rate } \\
\text { of change* }\end{array}$ & Pvaluet \\
\hline $00-24$ & 0.000 & 0.12 & 0.000 & 0.23 \\
\hline $25-34$ & 0.000 & 0.88 & 0.000 & 0.63 \\
\hline $35-44$ & +0.014 & 0.0005 & +0.011 & $<0.0001$ \\
\hline $45-54$ & +0.113 & $<0.0001$ & +0.048 & $<0.0001$ \\
\hline $55-64$ & +0.396 & $<0.0001$ & +0.152 & $<0.0001$ \\
\hline $65-74$ & +0.998 & $<0.0001$ & +0.501 & $<0.0001$ \\
\hline $75 \cdot 84$ & +1.777 & $<0.0001$ & +1.079 & $<0.0001$ \\
\hline $85+$ & +2.495 & $<0.0001$ & +1.811 & $<0.0001$ \\
\hline ASDR & +0.135 & $<0.0001$ & +0.069 & $<0.0001$ \\
\hline
\end{tabular}

- Per 100,000 of population per year: $+P$ value associated with the null hypothesis that rate of change is O. ASDR Age standardized mortality rate

TABLE 4

Age specific morbidity (1982) and mortality (1985) rates by age group and sex

\begin{tabular}{lcccccc}
\hline & \multicolumn{2}{c}{ Males } & & \multicolumn{2}{c}{ Females } \\
\cline { 2 - 3 } Age group (years) & $\begin{array}{c}\text { Morbidity } \\
\text { rate }\end{array}$ & $\begin{array}{c}\text { Mortality } \\
\text { rate }\end{array}$ & & $\begin{array}{c}\text { Morbidity } \\
\text { rate }\end{array}$ & $\begin{array}{c}\text { Mortality } \\
\text { rate }\end{array}$ \\
\hline $00-24$ & 0.06 & 0.00 & & 0.02 & 0.00 \\
$25-34$ & 0.37 & 0.21 & & 0.28 & 0.18 \\
$35-44$ & 2.03 & 1.97 & & 1.22 & 1.30 \\
$45-54$ & 10.89 & 8.42 & & 6.19 & 4.67 \\
$55-64$ & 33.20 & 26.66 & & 16.84 & 16.56 \\
$65-74$ & 68.23 & 61.93 & & 43.43 & 41.15 \\
$75-84$ & 105.67 & 99.41 & & 67.61 & 68.93 \\
$85+$ & 119.01 & 133.24 & & 88.19 & 83.49 \\
\hline
\end{tabular}

Rates are per 100.000 population per year 


\section{DISCUSSION}

Using the model proposed by Zdeb (9), the probability that a Canadian will die from carcinoma of the pancreas is $1.27 \%$ in males and $1.23 \%$ in females. Although this probability is low, particularly when compared to probabilities for dying from other neoplastic diseases, pancreatic carcinoma is consistently ranked among the 10 leading causes and sites of cancer in Canada for many age groups for both males and females, and accounts for at least $6 \%$ of all deaths from cancer for many age-sex groups (3).

The similarity between morbidity and mortality rates in Table 4 (most recent years for which incidence and mor tality rates are available) is reflective of the low survival rate among patients afflicted with this neoplastic disease. Five-year survival rates based on a large series of patients from England and Wales indicates that survival is most likely in males and females age 35 to 44 , with rates of 7 and $6 \%$, respectively. Survival rates are $4 \%$ or less for all other age groups (5).

The marked increase in mor tality noted for Canada in this study is characteristic of the pattern of this disease in other parts of the world. In England and Wales, mortality rates have risen from 2.9 to 10.6 deaths per 100,000 population per year in males during $1911-15$ to $1976-78$ and from 2.9 to 8.6 deaths per 100,000 population per year in females.

Inspection of age-sex-specific mortality rates indicates that dramatic increases in mortality rates have occurred for older members of the population. Rates have risen from 12.6 and 12.1 deaths per 100,000 population in males and females aged 75 years and older (1911-15) to 94.1 and 66.9 deaths per 100,000 population

\section{REFERENCES}

1. Causes of Death: Provinces by Sex and Canada by Sex and Age, Detailed Categories of the International Classification of Diseases (ICD). Catalogue 84-203 (Annual). Ottawa: Statistics Canada.

2. Cancer in Canada. Catalogue 82-207 (Annual). Ottawa: Statistics Canada.

3. Cancer Incidence and Mortality at Different Ages, Canada, 1975-1979. Ottawa: Statistics Canada, 1984.

4. Holman D, Armstrong B. Cancer mortality trends in Australia, 1910-1979,
(1976-78). Equally impressive increases are noted for males and females aged 45 to 54.55 to 64 and 65 to 74 where rates have risen by factors of approximately 3 or more and 2 or more, respectively.

Further analysis of British mortality rates by metropolitan counties and rural and intermediate regions failed to produce any major difference in mortality between rural and urban dwellers in either males or females. Similarly, analysis by social class failed to produce any dramatic differences between the five major classes for males and (married) females.

The epidemiology of pancreatic carcinoma in Australia has followed a similar course. Holman and Armstrong (4) found mortality rates in males to have increased more than fourfold (1.84 to 7.84 deaths per 100,000 population per year) and in females by threefold ( 1.57 to 4.58 deaths per 100,000 population per year).

Incidence rates around the world vary within and between nations (10). In Canada, rates in males vary from 3.7 (NorthWest Territories and Yukon) to 11.0 (Ontario) new cases per 100,000 population per year and between 4.1 (Newfoundland) and 11.5 (North West Territories and Yukon) new cases per 100,000 population per year in females. Similar variations are present in the United States between states and various ethnic communities where rates in Blacks are as much as double those noted in Whites rates for Chinese and Japanese Americans and American Indians are appreciably lower. In Asia, Jews of Israeli origin have the highest rate for males and one of the highest rates for females. Rates in India are among the lowest

Parts 1 and 2. Cancer Council of Western Australia, 1982.

5. Cancer statistics: Incidence, survival, and mor tality in England and Wales. Studies on medical and population subjects, No. 43. Office of population censuses and surveys. London: Cancer Research Campaign, 1981.

6. Segi M, Tominaga S, Aoki K, Fujimoto I. Cancer mortality and morbidity statistics. Japan and the world. GANN Monograph on Cancer Research, No. 26. Japanese Cancer Association. Tokyo: Japan Scientific Societies Press, rates in Asia and the world for both males and females. In Europe, rates are greatest in Cracow, Poland and Eastern Scotland where rates for males exceed 10 new cases per 100,000 population per year and Denmark, Norway, Sweden and Finland where rates for males exceed 8 new cases per 100,000 population per year and 5 new cases per 100,000 population per year in females.

Holman and Armstrong (4) note that the dramatic rise in pancreatic carcinoma is perhaps a reflection of both crosssectional and cohort-based effects and may involve an improvement in the ability to diagnose the disease. However, improvements in the reporting and/or diagnosis of this neoplastic disease is not totally supported by Canadian rates (Table 1) since 1970 as, for example, standardized incidence rates have been relatively stable during the period 1970-82 for males whereas rates in females have continued to rise. Fur thermore, the relative consistency in mortality rates between males and females $(50 \%$ to $70 \%$ higher rates in males) during the period studied suggests that potential exogenous risk factors are affecting males and females equally and/or that both sexes are exposed similarly.

Whatever the reasons, the increased mortality in Canada and many other regions of the world is of major concern, particularly in light of the fact that survival is low in afflicted patients and that many industralized societies have ageing populations which, coupled with the rapid rise in mortality for older individuals, should further escalate the absolute number of deaths noted in many societies, even if age standardized mortality rates stabilize.

1981.

7. Ayiomamitis A. The epidemiology of cancer of the uterine cervix in Canada: 1931 to 1984. Am J Obstet Gynecol 1987; 156:1075-80.

8. Ayiomamitis A. The epidemiology of cancer of the prostate in Canada: 1950-1984. Br J Urol 1987;60: 157-61.

9. Zdeb MS. The probability of developing cancer. Am J Epidemiol 1977;106:6-16.

10. Waterhouse J, Muir C, Shanmugaratnam K. Powell J. Cancer in five continents, Vol IV. Lyon: IARC Scientific Publication, 1982. 


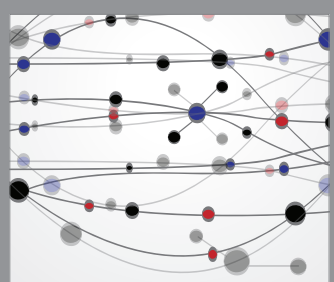

The Scientific World Journal
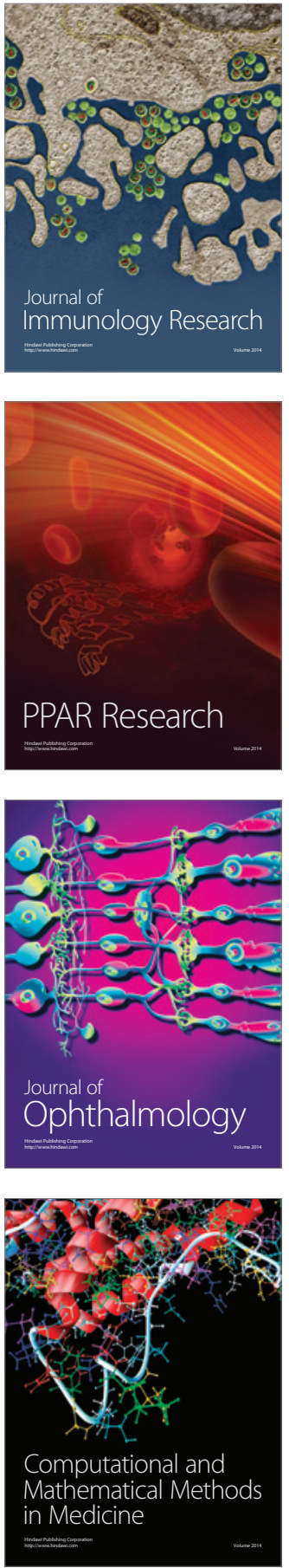

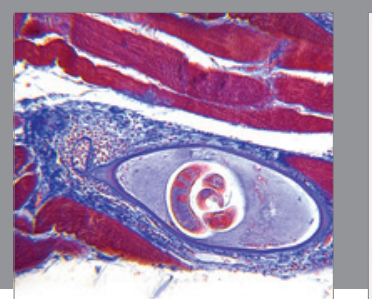

Gastroenterology Research and Practice

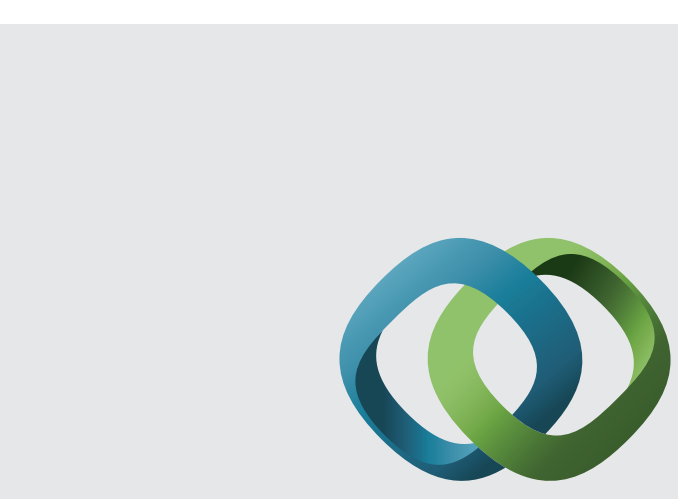

\section{Hindawi}

Submit your manuscripts at

http://www.hindawi.com
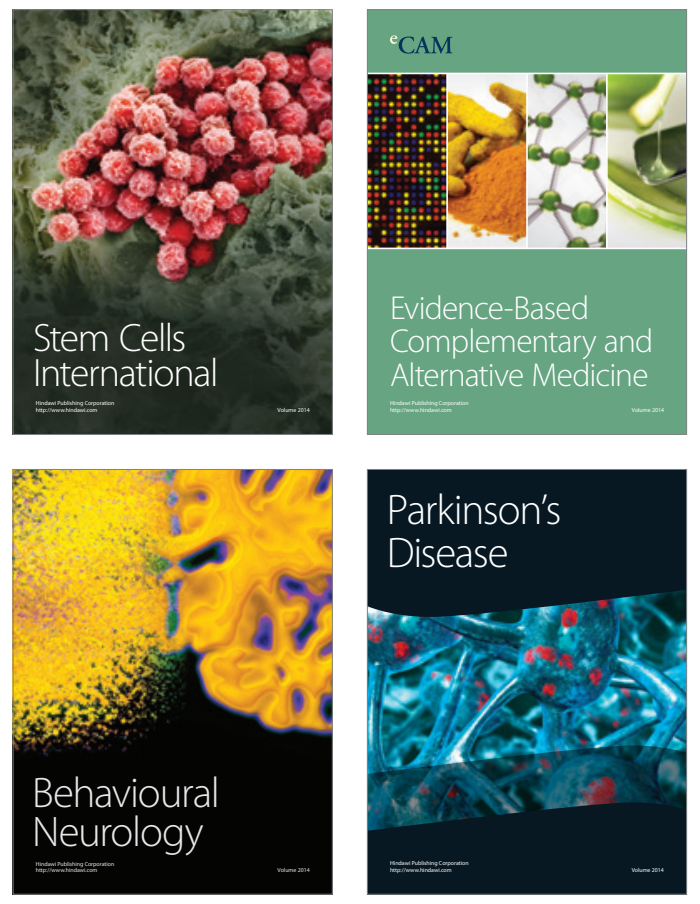
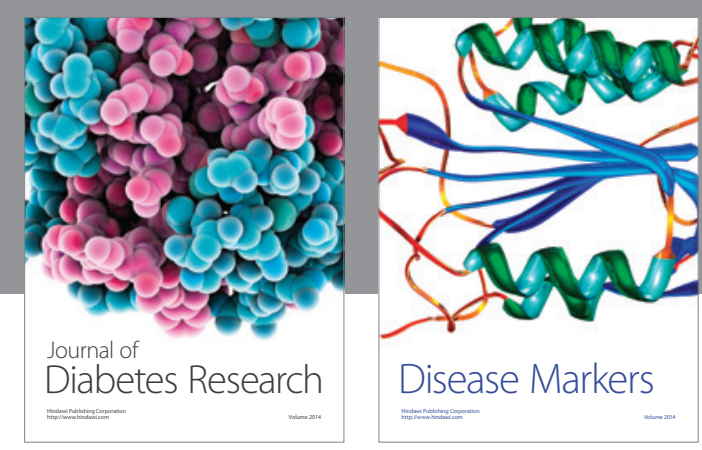

Disease Markers
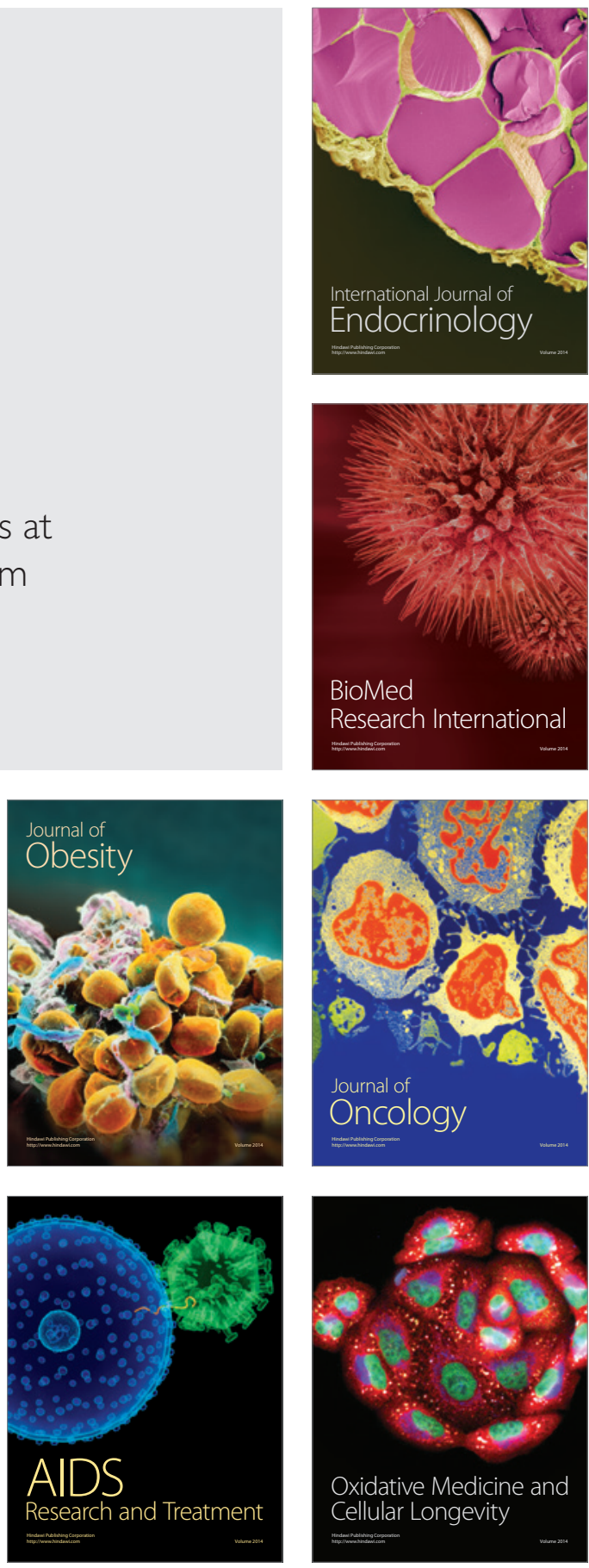